\title{
Tacrolimus-Induced
}

\section{Leukoencephalopathy in a Renal Transplantation Patient}

\author{
Ismail Ibrahim Ismail ${ }^{a}$ Ehab A. Abdelnabi ${ }^{b}$ Riyad Khan ${ }^{a}$ \\ Jasem Y. Al-Hashel ${ }^{a, c}$ Khaja M. Sharfuddin ${ }^{a}$ \\ ${ }^{a}$ Department of Neurology, Ibn Sina Hospital, Kuwait, Kuwait; bepartment of Radiology, \\ Ibn Sina Hospital, Kuwait, Kuwait; ' Department of Medicine, Faculty of Medicine, Health Sciences Centre, \\ Kuwait University, Kuwait, Kuwait
}

\section{Keywords}

Tacrolimus · Encephalopathy · Renal · Transplantation · Magnetic resonance imaging

\begin{abstract}
Tacrolimus is an immunosuppressant that is frequently used following renal transplantation. Several mild neurological side effects of tacrolimus have been reported in the literature; however, severe complications in the form of confusion, seizures, and coma are rare. Herein, we report a 16-year-old boy on tacrolimus following living-donor kidney transplant, who presented with subacute onset of hand tremors, headache, altered mental status, and progressed to akinetic mute state. $\mathrm{He}$ was diagnosed with tacrolimus-induced leukoencephalopathy based on findings of his magnetic resonance imaging and ruling out other possible causes. He showed clinical and radiological improvement after discontinuation of tacrolimus. Radiological features of tacrolimus-induced leukoencephalopathy are more heterogenous than commonly perceived and
\end{abstract}

karger@karger.com www.karger.com/dmj

Karger"
(C) 2022 The Author(s)

Published by S. Karger AG, Basel

This is an Open Access article licensed under the Creative Commons Attribution-NonCommercial-4.0 International License (CC BY-NC) (http://www.karger.com/Services/OpenAccessLicense), applicable to the online version of the article only. Usage and distribution for commercial purposes requires written permission. should be suspected in such patients. The potential neurotoxicity of tacrolimus should be recognized in patients with renal transplantation, and switching to a different immunosuppressant is warranted to prevent permanent neurological damage.

(c) 2022 The Author(s).

Published by S. Karger AG, Basel

\section{Introduction}

Tacrolimus is a calcineurin inhibitor that has been frequently used as an immunosuppressant after organ transplantation. It has been estimated that between $25 \%$ and $31 \%$ of patients who receive tacrolimus can experience neurotoxic side effects $[1,2]$. Most of these side effects are mild and tend to present shortly after transplantation. The risk of neurotoxicity from tacrolimus may vary according to the type of the organ transplanted, being more common in liver transplantation than in heart or lung transplantation, and they are rarely reported in renal transplantation patients [3]. 
Fig. 1. MRI study of the brain of a 16-yearold male; axial FLAIR (a-c), axial T2weighted images (d) showing supra and infratentorial white matter signal abnormalities involving: both cerebral hemispheres with nearly symmetrical appearance at both deep and subcortical white matter, more obvious at the anterior aspect of both temporal lobes, The same white matter hyperintensities involved the midbrain, pons, middle cerebellar peduncles. The deep grey matter nuclei including the basal ganglia and dentate nuclei were also involved by the signal abnormalities. Both thalami, hypothalamic regions, claustrum were spared bilaterally, and there was no diffusion restriction on DWI or abnormal enhancement (not shown). FLAIR, fluid-attenuated inversion recovery.
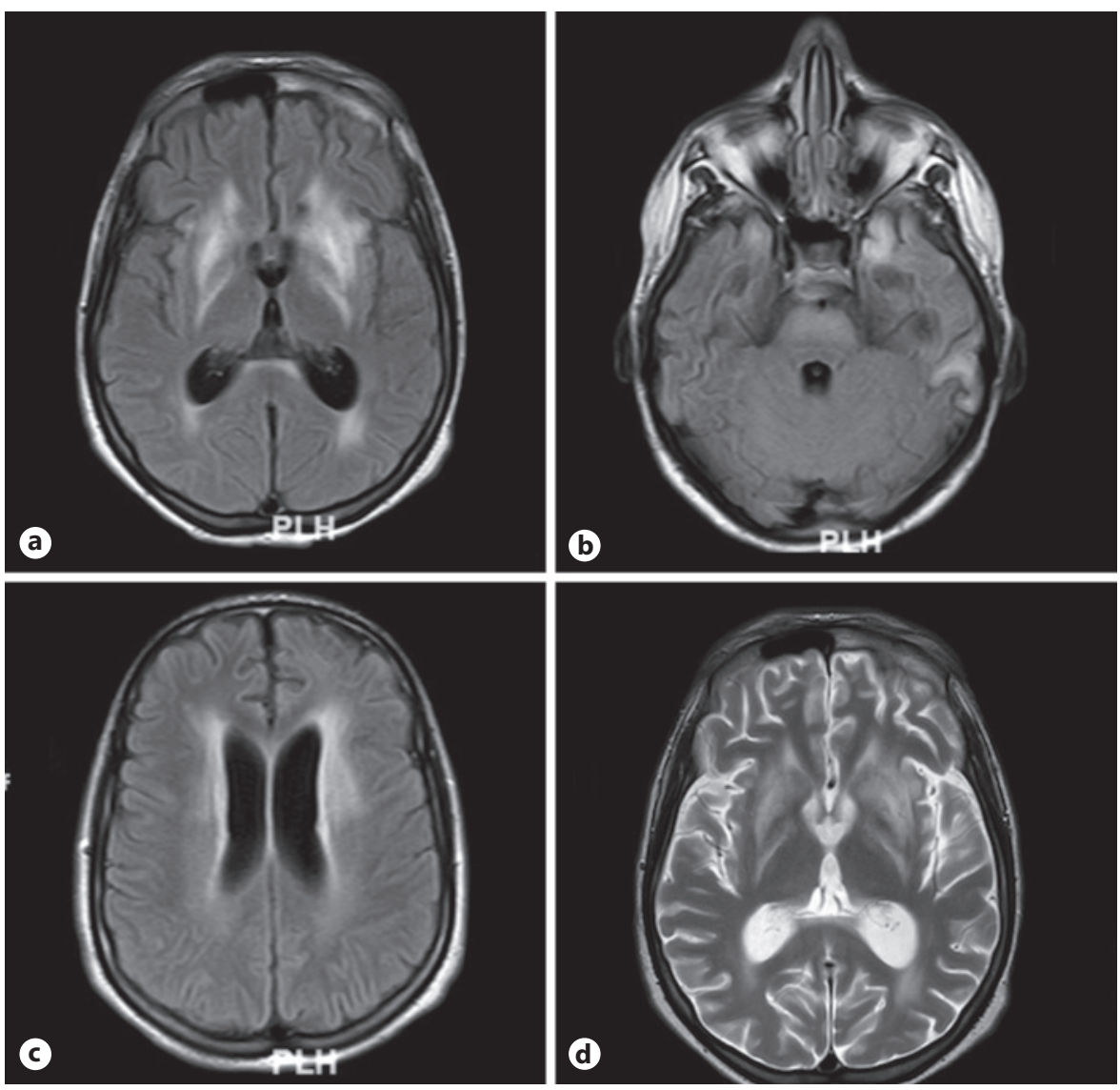

Tacrolimus-induced leukoencephalopathy is a rarely encountered phenomenon in patients with renal transplantation. The commonest described clinical entity is posterior reversible leukoencephalopathy syndrome; however, several studies have shown that tacrolimus-induced leukoencephalopathy is not a homogenous entity, and atypical imaging characteristics can be challenging in such cases [4]. Herein, we describe a rare case of tacrolimus-induced leukoencephalopathy following renal transplantation, with affection of multiple regions of the brain on magnetic resonance imaging (MRI) that improved after discontinuation of the drug.

\section{Case Report}

A 16-year-old boy, who is a living-donor renal transplantation recipient, presented with subacute onset of hand tremors, headache, and gait difficulty of 1-week duration that progressed within few days to disorientation, confusion, and akinetic mute state. $\mathrm{He}$ had no history of fever, nor previous similar clinical condition. $\mathrm{He}$ was maintained on oral tacrolimus ( $5 \mathrm{mg}$ twice daily), predniso- lone (60 $\mathrm{mg}$ per day), and mycophenolate mofetil ( $2 \mathrm{~g}$ per day) for 6 months after renal transplantation. Neurological examination showed disorientation to time, person, and place, with receptive and expressive dysphasia, bilateral rigidity, hyperreflexia, and bilateral extensor plantar response. MRI of the brain showed bilateral diffuse T2-weighted/fluid-attenuated inversion recovery hyperintensities involving frontal, parietal, temporal lobes, corpus callosum, as well as mesencephalon and rhombencephalon, and cerebellar peduncles (Fig. 1).

An extensive laboratory workup was performed to rule out metabolic, infectious, autoimmune, and paraneoplastic causes, which all yielded negative results. Cerebrospinal fluid showed normal protein, glucose, cell count and differential, negative bacterial, fungal and mycobacterial cultures, and negative PCR for HSV, CMV, EBV, VZV, EV, ADV, HHV 6, 7, 8, and JCV. Electroencephalography showed diffuse slowing, with no epileptiform discharges. Tacrolimus trough level was within therapeutic range, 9.7 $\mathrm{ng} / \mathrm{mL}$ (normal values: $5.0-15.0$ ).

The diagnosis of tacrolimus-induced leukoencephalopathy was made, and the drug was stopped. He was treated with intravenous methylprednisolone, followed by oral steroid therapy. The patient showed clinical improvement as regards to consciousness and rigidity. His tremors disappeared and he became fully ambulant. A follow-up MRI showed complete radiological improvement after discontinuation of tacrolimus. 


\section{Discussion}

Tacrolimus is an immunosuppressive agent that has been used effectively for prevention of organ transplant rejection. It is a macrolide that belongs to the family calcineurin inhibitors, which suppresses the immune system by inhibiting calcineurin phosphatase, and subsequently, inhibit calcineurin and T-cell activation [5]. The clinical and radiological features of tacrolimus-associated leukoencephalopathy are variable and pose a diagnostic challenge.

Several neurological complications have been reported with tacrolimus intake, irrespective of tacrolimus levels. It has been shown that serum drug trough levels of tacrolimus are poorly correlated with the occurrence of neurotoxicity [6]. Most symptoms are mild, including paresthesia, tremors, headache, and dizziness. Fine tremors of upper limbs are the most pronounced neurological complication and can help in early diagnosis [7].

However, severe complications in the form of confusion, seizures, psychosis, cortical blindness, and coma are rare, affecting $1-6 \%$ of transplant recipients. Neurotoxicity is more common early after initiation of the drug, but can happen months or years later [8].

The exact mechanism by which tacrolimus causes neurotoxicity remains poorly understood, and most probably, multifactorial. The drug has high lipophilicity, which facilitates its passage through blood-brain barrier to the brain, leading to cerebral vasodilatation and vasogenic edema [9]. Moreover, it can exert a direct neurotoxic effect, cytotoxic edema, direct endothelial damage, vasoconstriction, demyelination, or inhibition of the expression of drug-efflux pumps [10]. Radiologically, although the commonest presentation is described under the term of posterior reversible encephalopathy syndrome, several MRI studies have shown that imaging characteristics are variable and more heterogenous than commonly perceived [11]. Wu et al. [12] reported 5 patients with 9 clinical episodes where the most commonly involved region was the parieto-occipital region, which was seen in all 9 episodes. This was followed by the frontal lobe in 6, deep white matter in 4 , cerebellum in 3 , basal ganglia in 2 , brainstem in 2, and the thalamus and temporal lobe in 1 episode, respectively. Moreover, in a prospective study on 14 patients receiving tacrolimus who developed neurologic complications, 5 patients had abnormalities involving the white matter, 1 had putaminal hemorrhage, and 8 were unremarkable, on brain MRI [13].

Differential diagnosis of leukoencephalopathy after kidney transplantation is wide and requires a high level of suspicion. Several etiologies could be implicated including infections (e.g., bacterial, viral, fungal, mycobacteria), toxic or metabolic encephalopathy, demyelinating disorders, intracranial vasculitis, progressive multifocal leukoencephalopathy, X-linked adrenoleukodystrophy, cerebral venous thrombosis, acute disseminated encephalomyelitis, and encephalopathy of unknown origin [14, $15]$.

\section{Conclusion}

Early recognition of tacrolimus-induced neurotoxicity can be challenging. Our case demonstrates that leukoencephalopathy during tacrolimus therapy can occur with therapeutic drug level and can involve different regions of the brain. Tacrolimus neurotoxicity should be considered in the differential diagnosis of T2-weighted, and fluid-attenuated inversion recovery hyperintensities on MRI in patients with renal transplantation, in addition to infectious, neoplastic/paraneoplastic, and autoimmune causes. Drug stoppage and replacement by another immunosuppressive agent can ameliorate the neurological manifestations, significantly. Discontinuation of tacrolimus should be considered as a therapeutic trial, since the diagnosis can be confirmed by improvement after removal of the drug.

\section{Acknowledgments}

We would like to thank the patient and his family for their cooperation.

\section{Statement of Ethics}

Our institution does not require ethical approval for reporting individual cases or case series. The patient has given a written consent for publication of this case report and the MRI-images attached.

\section{Conflict of Interest Statement}

The authors have no conflicts of interest to disclose.

\section{Funding Sources}

Funding sources are not applicable for this study. 


\section{Author Contributions}

I.I.I. drafted the manuscript and performed literature review; E.A.A. analyzed and reported M.R. images; R.K. and J.Y.A. critically revised the manuscript for intellectual content; K.M.S. was the treating neurologist, collected clinical data on the patient, and revised the manuscript. The author(s) read and approved the final manuscript.

\section{References}

1 Eidelman BH, Abu-Elmagd K, Wilson J, Fung JJ, Alessiani M, Jain A, et al. Neurologic complications of FK 506. Transplant Proc. 1991; 23:3175-8.

2 Wijdicks EF, Wiesner RH, Dahlke LJ, Krom RA. FK506-induced neurotoxicity in liver transplantation. Ann Neurol. 1994;35:498501.

3 Singh N, Bonham A, Fukui M. Immunosuppressive-associated leukoencephalopathy in organ transplant recipients. Transplantation. 2000;69:467-72.

4 Lavigne CM, Shrier DA, Ketkar M, Powers JM. Tacrolimus leukoencephalopathy: a neuropathologic confirmation. Neurology. 2004; 63:1132-3.

5 Hamawy MM. Molecular actions of calcineurin inhibitors. Drug News Perspect. 2003 Jun; 16(5):277-82.

6 Wijdicks EF. Neurotoxicity of immunosuppressive drugs. Liver Transpl. 2001 Nov 1; 7(11):937-42.

\section{Data Availability Statement}

All data generated or analyzed during this study are included in this article. Further inquiries can be directed to the corresponding author.
7 Small SL, Fukui MB, Bramblett GT, Eidelman BH. Immunosuppression-induced leukoencephalopathy from tacrolimus (FK506). Ann Neurol. 1996;40:575-80.

8 Pflugrad H, Nösel P, Ding X, Schmitz B, Lanfermann $\mathrm{H}$, Barg-Hock $\mathrm{H}$, et al. Brain function and metabolism in patients with longterm tacrolimus therapy after kidney transplantation in comparison to patients after liver transplantation. PloS One. 2020 Mar 10; 15(3):e0229759.

9 Bechstein WO. Neurotoxicity of calcineurin inhibitors: impact and clinical management. Transpl Int. 2000;13:313-26.

10 Saadi A, Schmahmann JD. Pearls \& oy-sters: tacrolimus neurotoxicity presenting as an isolated brainstem lesion. Neurology. 2016; 86(11):e109-11.
11 Furukawa M, Terae S, Chu B, Kaneko K, Kamada $\mathrm{H}$, Miyasaka K. MRI in seven cases of tacrolimus (FK-506) encephalopathy: utility of FLAIR and diffusion-weighted imaging. Neuroradiology. 2001;43:615-21.

12 Wu Q, Marescaux C, Qin X, Kessler R, Yang $\mathrm{J}$. Heterogeneity of radiological spectrum in tacrolimus-associated encephalopathy after lung transplantation. Behav Neurol. 2014 Jan 1;2014:931808.

13 Shimono T, Miki Y, Toyoda H, Egawa H, Uemoto $\mathrm{S}$, Tanaka $\mathrm{K}$, et al. MR imaging with quantitative diffusion mapping of tacrolimus-induced neurotoxicity in organ transplant patients. Eur Radiol. 2003;13:986-93.

14 Lee VH, Wijdicks EF, Manno EM, Rabinstein AA. Clinical spectrum of reversible posterior leukoencephalopathy syndrome. Arch Neurol. 2008;65(2):205-10.

15 Cicora F, Roberti J. Progressive multifocal leukoencephalopathy in a kidney transplant recipient. Exp Clin Transplant. 2019 Feb; 17(1):108-10. 